Pacific

Journal of

Mathematics

\title{
A GIAMBELLI-TYPE FORMULA FOR SUBBUNDLES OF THE TANGENT BUNDLE
}

BORIS SHAPIRO AND MAXIM KAZARIAN 


\title{
A GIAMBELLI-TYPE FORMULA FOR SUBBUNDLES OF THE TANGENT BUNDLE
}

\author{
BORIS SHAPIRO AND MAXIM KAZARIAN
}

\begin{abstract}
Consider a generic $\boldsymbol{n}$-dimensional subbundle $\mathscr{V}$ of the tangent bundle $\boldsymbol{T M}$ on some manifold $M$. Given $\mathscr{V}$, one can define different degeneracy loci $\Sigma_{r}(\mathscr{V}), r=\left(r_{1} \leq r_{2} \leq r_{3} \leq \cdots \leq r_{k}\right)$, on $M$ consisting of all points $x \in M$ for which the subspaces $\mathscr{V} j(x) \subset T M(x)$ spanned by all length $\leq j$ commutators of vector fields tangent to $\mathscr{V}$ at $\boldsymbol{x}$ has dimension less than or equal to $\boldsymbol{r}_{j}$. Under a certain transversality assumption, we explicitly calculate the $\mathbb{Z}_{2}$ cohomology classes of $M$ dual to $\Sigma_{r}(\mathscr{V})$, using determinantal formulas due to $W$. Fulton and the expression of the Chern classes of the associated bundle of the free Lie algebras in terms of the Chern classes of $\mathscr{V}$.
\end{abstract}

\section{Preliminaries and results}

History and motivation. The question of the existence of a nontrivial subbundle of the tangent bundle on a given manifold is a geometric problem of long-standing interest. (Such subbundles are often called distributions, and we will freely use both terms below.) In the basic nontrivial case of rank-2 subbundles, the first important results in the area go back to the classical treatise [Hirzebruch and Hopf 1958]. Apparently, the best achievements in this problem were obtained in late 1960s by Thomas [1967a; 1967b]; see also the well-written survey [Thomas 1969]. Later, some of his results were rediscovered by Matsushita [1988]. Not much has been done in this area since then. One of the few recent exceptions is [Jacobowitz and Mendoza 2003]. Rather detailed information is available about the existence of (oriented) subbundles of rank 2. For rank 3 and higher, only the first obstruction to the problem is known, see [Thomas 1967a]. The algebraic invariants for this result come from the Stiefel-Whitney classes of elements of $\mathbb{K} \tilde{\mathbb{O}}(M)$, which is the reduced real $K$-theory group of the manifold. Starting with the late 1970s, the interest in the geometric properties of subbundles of the tangent bundle were stimulated by the development of singularity theory and the revival of interest in nonholonomic mechanics. A nice source of information about this topic is [Montgomery 2002]. In particular, if a given manifold admits a subbundle of rank at least

MSC2000: 57R20, 57R22.

Keywords: $n$-subbundles, free Lie algebra, determinantal formulas. 
2 , one can construct at each point of the manifold an incomplete flag whose $i$-th subspace is the linear span of the commutators of length at most $i$ of the vector fields tangent to the subbundle. The ranks of these subspaces will (in general) depend on the point; see below.

For a small generic perturbation of the original subbundle, the ranks of the subspaces of the incomplete flags will stay constant almost everywhere on the manifold, and depend only on the rank of the original subbundle and the dimension of the manifold. Generalizing the question with which we started, one can formulate this problem:

Problem. When does a manifold admit a distribution whose associated flags have constant (and maximal-possible) ranks throughout the manifold?

Being in general even more difficult than the (still unsolved) initial question, the latter problem has a nice answer in the case of oriented rank-2 distributions on oriented 4-manifolds. A rank-2 distribution on a 4-manifold whose associated flag has the set of ranks $(2,3,4)$ at each point is called an Engel distribution. These distributions have remarkable properties; see example [Gershkovich 1995a; 1995b]. For instance, combining the results of this second paper (see also [Kazarian et al. 1997]) with the recent [Vogel 2004; 2005], we get:

Theorem. An orientable 4-manifold admits an orientable Engel distribution if and only if it is parallelizable.

The aim of this paper is to develop the basics of the obstruction theory suited to the problem above.

Standard definitions and notation. The following notions are standard, see for example [Gershkovich and Vershik 1988]. Let $M$ be an $m$-dimensional manifold and $\mathscr{V} \subset T M$ an $n$-dimensional subbundle (a rank $n$-distribution) on $T M$. Given $\mathscr{V}$, one associates at each point $x \in M$ its derived flag

$$
f \mathscr{V}_{x}=\left\{\mathscr{V}_{x}=\mathscr{V}_{x}^{1} \subseteq \mathscr{V}_{x}^{2} \subseteq \cdots \subseteq \mathscr{V}_{x}^{j} \subseteq \cdots\right\},
$$

where $\mathscr{V}_{x}^{j}=\left\{\mathscr{V}_{x}^{j-1}+\left[\mathscr{V}^{j-1}, \mathscr{V}\right]\right\}_{x}$. If at each point $x \in M$ there exists a positive integer $k(x)$ such that the subspace $\mathscr{V}_{x}^{k(x)}$ coincides with $T M_{x}$, then $\mathscr{V}$ is called (maximally) nonholonomic. Let $n_{j}(x)$ denote the dimension of $\mathscr{V}_{x}^{j}$. The set of numbers $\left(n_{1}(x), \ldots, n_{k}(x)\right)$ is called the growth vector of $\mathscr{V}$ at $x$. For a given nonholonomic $\mathscr{V}$, the minimal number $k$ such that $\mathscr{V}_{x}^{k}=T_{x} M$ at all points $x$ is called the degree of nonholonomicity. (A subbundle $\mathscr{V}$ is called regular if the $n_{j}(x)$ do not depend on $x$; the corresponding set of numbers $\left(n_{1}, n_{2}, \ldots, n_{k}\right)$ is called the growth vector of the regular subbundle $\mathscr{V}$.) Let $\mathfrak{L}_{n}$ denote the free Lie algebra with $n$ generators, and let $\mathfrak{L}_{n}^{k}$ be its linear subspace spanned by all elements of length $k$. Let $d(n, k)$ be the dimension of $\mathfrak{L}_{n}^{k}$. An $n$-dimensional $\mathscr{V}$ with degree of 
nonholonomicity $k$ is called a maximal-growth subbundle (or an MG-distribution) if $n_{k}=m$ and $n_{j}=\sum_{i \leq j} d(n, j)$ for all $j<k$. The growth vector with the entries

$$
(n=d(n, 1), d(n, 1)+d(n, 2), \ldots, d(n, 1)+d(n, 2)+\ldots+d(n, k), m)
$$

is called the maximal growth vector.

Remark. According to [Gershkovich 1988], a germ of a generic distribution has maximal growth. Here, generic means belonging to some open everywhere-dense subset in the $C^{\infty}$-Whitney topology. Thus, locally a typical subbundle is an MGdistribution, while globally there are (many) topological obstructions to the existence of MG-distributions on a given $M$. The problem we are addressing in the present paper can be reformulated as constructing obstructions to the existence of MG-distributions on a given manifold.

Examples. A contact structure is a regular MG-distribution. A 2-dimensional MG-subbundle on a four-dimensional manifold is called an Engel distribution; see above and [Gershkovich 1995b]. This is the only example of distributions with a stable local normal form, besides contact structures and their even-dimensional analogs.

Degeneracy loci. Given a generic $n$-distribution $\mathscr{V} \subset T M$, one expects that globally $M$ contains a (typically, reducible) degeneracy locus $\Sigma$ consisting of all such points $x$ where the growth vector $\left(n_{1}(x), \ldots, n_{k}(x)\right)$ is lexicographically smaller than the maximal one. Given a growth vector $r=\left(r_{1} \leq r_{2} \leq r_{3} \leq \cdots \leq r_{k}\right)$, denote by $\Sigma_{r}$ the subset of all $x \in M$ satisfying the conditions $n_{1}(x) \leq r_{1}, \ldots, n_{k}(x) \leq r_{k}$. Such $\Sigma_{r}$ can be considered as degeneracy loci in the standard meaning of intersection theory; see Section 2 (compare with [Fulton 1984, Chapter 14]). Namely, each $n$-distribution $\mathscr{V} \subset T M$ induces the associated fiber bundle $\mathfrak{L}(\mathscr{V}) \rightarrow M$, where the fiber $\mathfrak{L}_{x}$ is the free Lie algebra generated by the subspace $\mathscr{V}_{x}$. The bundle $\mathfrak{L}(\mathscr{V})$ has an obvious grading $\mathfrak{L}(\mathscr{V})=\bigoplus_{k=1}^{\infty} \mathfrak{L}^{k}(\mathscr{V})$ coming from $\mathfrak{L}_{n}=\bigoplus_{k=1}^{\infty} \mathfrak{L}_{n}^{k}$. Moreover, one can define a natural map $\Phi: \mathfrak{L}(\mathscr{V}) \rightarrow T M$ of vector bundles, sending each $\bigoplus_{i=1}^{k} \mathfrak{L}^{i}(\mathscr{V})$ onto $\mathscr{V}^{k}$. (The map $\Phi$ is not unique, in very much the same way as the identifying map between $T M$ and its nilpotentization; compare with [Gershkovich and Vershik 1988].) This allows us to apply the determinantal formulas of [Fulton 1992] to the map $\Phi$, under the assumption that the considered $\Sigma_{r}$ has the expected (co)dimension, that is, the same codimension as the corresponding degeneracy locus for a generic map of flag bundles of the dimensions prescribed by $\mathfrak{L}_{n}$. Algebraically, in order to be able to apply these formulas one also needs to express the Chern classes of $\mathfrak{L}(\mathscr{V})$ in terms of those of $\mathscr{V}$.

Remark. Fulton [1992] has generalized a large number of previously known special cases of determinantal formulas giving the cohomology classes of different 
degeneracy loci for the maps of vector bundles to a very general situation of maps of flagged vector bundles. Such formulas could be traced back (through the works of Porteous, Thom, Laksov and Kempf, and many other authors) to the pioneering results of Giovanni Giambelli on the degrees of different strata in the spaces of matrices. For this reason in a number of publications similar determinantal formulas are named after Giambelli; see, for example, [Fulton 1992, §7]. For a detailed account on determinantal formulas and degeneracy loci we recommend [Fulton and Pragacz 1998]; for information on Giambelli, see [Laksov 1994].

The contents of the paper are as follows. In Section 2 we construct the map $\Phi: \mathfrak{L}(\mathscr{V}) \rightarrow T M$. In Section 3 we find an explicit formula for the Chern character of the bundle $\mathfrak{L}^{k}(\mathscr{V})$ in terms of the Chern character of $\mathscr{V}$; it turns out to be similar to the formula for the dimensions of $\mathfrak{L}_{n}^{k}$. In principle, this allows us to calculate the Chern classes of $\mathfrak{L i e}^{k}(\mathscr{V})$ for any reasonable specific example by inverting Newton polynomials; see the Appendix. In Section 4 we recall the appropriate determinantal formula for maps of flag bundles, adjust it to our needs and calculate some examples. Section 5 is devoted to some generalities on derived flags $f \mathscr{V}$ and the standard stratification of the spaces of matrices as well as counterexamples to transversality in big codimensions. In Section 6 we enumerate all potentially admissible defect vectors occurring for a generic distribution and prove the necessary transversality result showing that $\Sigma_{r}$ have the expected (co)dimension in the cases $n=2, m \leq 8$ and $n \geq 3, m \leq \frac{1}{6} n(n+1)(2 n+1)$. In Section 7 we briefly discuss some further directions of study and possible generalizations of the transversality theorem. The main result of the paper is formula (11) justified by the transversality theorem for the above mentioned values of $(n, m)$. Finally, appendix contains the Mathematica code which explicitly calculates the necessary Chern classes of the homogeneous components of the free Lie algebra $\mathfrak{L}_{n}^{k}$ up to order 4.

In short, the main proposition of the paper can be summarized as follows. In order to calculate the universal formula for the cohomology class dual to $\Sigma_{r}$ for a given growth vector $r$ one has to substitute the expressions for the Chern (or rather for the Stiefel-Whitney) classes of $\bigoplus_{i=1}^{k} \mathfrak{L}^{i}(\mathscr{V})$ and $T M$ into the appropriate Giambelli-type formula.

\section{The $\Sigma_{r}$ as classical degeneracy loci}

Associated bundle of free Lie algebras. Given $\mathscr{V} \subset T M$, we define the map $\Phi$ : $\mathfrak{L}(\mathscr{V}) \rightarrow T M$ such that each subbundle $\bigoplus_{i=1}^{j} \mathfrak{L}^{i}(\mathscr{V})$ is mapped onto $\mathscr{V}^{j}$. In fact we define another filtered vector bundle $N(\mathscr{V})$ whose associated graded bundle is isomorphic to $\mathfrak{L}(\mathscr{V})$ with the canonical map $\Psi: N(\mathscr{V}) \rightarrow T M$. As a result one gets a (nonunique) map $\Phi: \mathfrak{L}(\mathscr{V}) \rightarrow T M$ defined up to a filtered isomorphism between $N(\mathscr{V})$ and $\mathfrak{L}(\mathscr{V})$. 
Universal map. Let $\mathscr{V} \subset T M$ be an $n$-subbundle and $k$ be its degree of nonholonomicity.

Theorem 1. There exists a globally defined map of vector bundles

$$
\Phi_{k}: \bigoplus_{i \leq k} \mathfrak{L}^{i}(\mathscr{V}) \rightarrow T M
$$

such that for all $x \in M$ and $j \leq k$, the subspace $\mathscr{q}^{j}(x)$ coincides with the image of $\Phi_{j}(x): \bigoplus_{i \leq j} \mathfrak{L}^{i}(\mathscr{V})(x) \rightarrow T_{x} M$, where $\Phi_{j}$ is the restriction of $\Phi_{k}$ to $\bigoplus_{i \leq j} \mathfrak{L}^{i}(\mathscr{V}) \subset$ $\bigoplus_{i \leq k} \mathfrak{L}^{i}(\mathscr{V})$.

For the local version of this theorem, see [Gershkovich and Vershik 1988].

Proof. We construct an auxiliary flag of vector bundles

$$
N^{1}(\mathscr{V}) \subset N^{2}(\mathscr{V}) \subset \ldots N^{k}(\mathscr{V})
$$

the map $\Psi_{k}: N^{k}(\mathscr{V}) \rightarrow T M$ satisfying the statement of the Theorem, and a canonical isomorphism

$$
N^{j}(\mathscr{V}) / N^{j-1}(\mathscr{V}) \cong \mathfrak{L}^{j}(\mathscr{V})
$$

which shows that the flag of bundles $N(\mathscr{V})$ is isomorphic to the flag of bundles $\mathfrak{L}(\mathscr{V})$.

Recall the notion of a Hall basis in the free Lie algebra $\mathfrak{L}_{n}$ with $n$ generators, see [Bourbaki 1975]. Namely, $\mathfrak{L}_{n}$ has the following standard graded basis $H$ called Hall family or Hall basis. Given a linearly ordered set $V$ (of cardinality $n$ ), we define the following linearly ordered subset $H$ in the free monoid $M_{o} n_{V}$.

(1) If $u, v \in H$ and $\operatorname{lng}(u)<\operatorname{lng}(v)$ then $u<v$ where $\operatorname{lng}$ denotes the usual length of a word in $\mathrm{Mon}_{V}$.

(2) $V=H^{1} \subset H$ and $H^{2}$ consists of the set of all ordered pairs $\left(v_{1}, v_{2}\right) \in H$ where $v_{1}<v_{2}$.

(3) Each element of $H$ of length at least 3 has the form $a(b c)$, where $a, b, c \in H$, $b c \in H, b \leq a<b c$ and $b<c$. (Obviously, $H=\bigcup_{k=1}^{\infty} H^{k}$, where $H^{k}$ is the set of all length $k$ elements in $H$.)

Examples. Suppose $V=\{u<v\}$. Then

$$
\begin{aligned}
& H^{1}=\{u ; v\}, \quad H^{2}=\{(u, v)\}, \quad H^{3}=\{(u(u, v)) ;(v(u, v))\} \\
& H^{4}=\{(u(u(u, v))) ;(v(u(u, v))) ;(v(v(u, v)))\} \\
& H^{5}=\{(u(u(u(u, v)))) ;(v(u(u(u, v)))) ;(v(v(u(u, v)))) ; \\
& \quad(v(v(v(u, v)))) ;((u, v)(u(u, v))) ;((u, v)(v(u, v)))\} .
\end{aligned}
$$


The construction of the flag of bundles $N(\mathscr{V})$ is as follows. Let $W$ be the sheaf of free Lie algebras associated to the sheaf of local sections of the bundle $\mathscr{V} \subset$ $T M$. The elements of $W$ are $\mathbb{R}$-linear combinations of Lie monomials of sections of $\mathscr{V}$. Denote by $\mathscr{A}$ the sheaf of rings of smooth functions on $M$. Define the homomorphism $D: W \rightarrow$ derA as follows. If $v \in \mathscr{V} \subset W$ is of degree 1 we put

$$
D_{v} f=v f,
$$

i.e., the usual Lie derivative of the function $f$ along the vector field $v$. Then, we assign by induction

$$
D_{[a, b]} f=D_{b}\left(D_{a} f\right)-D_{a}\left(D_{b} f\right) .
$$

The operation $D$ is well defined and $D_{u}$ is the derivation of $\mathscr{A}$ for every $u \in W$. Consider the sheaf $\mathscr{A} \otimes_{\mathbb{R}} W$ of $\mathscr{A}$-modules. We introduce the Lie algebra structure on $\mathscr{A} \otimes W$ as

$$
[f \otimes u, g \otimes w]=f g \otimes[u, w]+f D_{u} g \otimes w-g D_{w} f \otimes u
$$

(the Jacobi identity is verified by direct computation). Finally, define $N(\mathscr{V})$ to be the quotient Lie algebra of $\mathscr{A} \otimes W$ by the ideal generated by all relations of the form

$$
f \otimes v=1 \otimes f v, \quad f \in \mathscr{A}, \quad v \in \mathscr{V} \subset W .
$$

Having (4) in mind we drop the sign of the tensor product in the notation of the elements in $N(\mathscr{V})$. The filtration on $W$ by the length of Lie monomials gives the natural filtration $N^{j}(\mathscr{V})$ on the sheaf $N(\mathscr{V})$ of $\mathscr{A}$-modules. We claim that all $N^{j}(\mathscr{V})$ are locally free sheaves of $\mathscr{A}$-modules of finite ranks. Indeed, let $e_{1}, \ldots, e_{n}$ be the set of local sections of $\mathscr{V}$ over some open domain $U \subset M$ such that these sections form a basis in each fiber $\mathscr{V}(x), x \in U$. Then it follows from (1)-(4) that every section $u$ of $W$ over $U$ can be represented as

$$
u=\sum f_{l} h_{l}\left(e_{1}, \ldots, e_{n}\right)
$$

where $f_{l}$ are some functions and $h_{l} \in H$ are the elements of Hall basis of the free Lie algebra $\mathfrak{L}_{n}$. Moreover, this representation is unique, i.e. the set of sections $h_{l}\left(e_{1}, \ldots, e_{n}\right), l \leq \operatorname{dim} \bigoplus_{i \leq j} \mathfrak{L}_{n}^{i}$ forms the set of free generators of the $\mathscr{A}$-module $N^{j}(\mathscr{V})$. Thus the $\mathscr{A}$-module $N^{j}(\mathscr{V})$ is the module of sections of some vector bundle which we also denote as $N^{j}(\mathscr{V})$.

Observe that if $[u, w] \in N(\mathscr{V})$ has degree $j$ then $[f u, g w]-f g[u, w]$ has degree strictly less than $j$. Therefore, the homomorphism $N^{j}(\mathscr{V}) / N^{j-1}(\mathscr{V}) \rightarrow \mathfrak{L}^{j}(\mathscr{V})$ is well defined. Moreover, the arguments above show that this homomorphism is, in fact, an isomorphism of vector bundles. 
The homomorphism of Lie algebras $\Psi: N(\mathscr{V}) \rightarrow \operatorname{Vect}(M)$ is now obvious. It sends a formal Lie bracket of vector fields in $\mathscr{V}$ to the corresponding commutator of these vector fields. Formulas (1)-(4) show that this homomorphism is well defined and $\Psi\left(N^{j}(\mathscr{V})(x)\right)$ coincides with $\mathscr{V}^{j}(x)$ by definition.

Remark. The vector bundle $N^{j}(\mathscr{V})$ can be also described, as a usual vector bundle by trivializations and transition functions. Trivializations of $N^{j}(\mathscr{V})$ correspond to the trivializations of $\mathscr{V}$ and are given by the sections $h_{l}\left(e_{1}, \ldots, e_{n}\right)$, $l \leq \operatorname{dim} \bigoplus_{i \leq j} \mathfrak{L}_{n}^{i}$, where $e_{1}, \ldots, e_{n}$ are sections giving some local basis of $\mathscr{V}$. If $\left\{e_{1}^{\prime}, \ldots, e_{n}^{\prime}\right\}$ is another basis such that $e_{i}=\sum a_{i r} e_{r}^{\prime}$ then to find transition functions for $N^{j}(\mathscr{V})$ one should express $h_{l}\left(\sum a_{1 p} e_{p}^{\prime}, \ldots, \sum a_{n p} e_{p}^{\prime}\right)$ using (1)-(4) as a linear combination of $h_{i}\left(e_{1}^{\prime}, \ldots, e_{n}^{\prime}\right)$ with some functional coefficients.

\section{On the Chern classes of the bundle of free Lie algebras}

Let $E \rightarrow M$ be a complex vector bundle of dimension $n$ over a smooth compact manifold $M$ (not necessarily a subbundle of $T M$ ). For any linear representation of the group $\operatorname{GL}(n, \mathbb{C})$ in $\mathbb{C}^{m}$ one can associate in a natural way to the bundle $E \rightarrow$ $M$ the corresponding $m$-dimensional bundle over $M$. For example, the bundles $E \otimes E, E^{*}, \Lambda^{2} E$ etc. are associated with the obvious representations of $\operatorname{GL}(n, \mathbb{C})$ in $\mathbb{C}^{n} \otimes \mathbb{C}^{n}, \mathbb{C}^{n *}, \Lambda^{2} \mathbb{C}^{n}$ respectively.

Given a basis $\left\{e_{1}, \ldots, e_{n}\right\}$ in $\mathbb{C}^{n}$ let $\mathfrak{L}_{n}$ denote the free Lie algebra with the generators $\left\{e_{1}, \ldots, e_{n}\right\}$, and let $\mathfrak{L}_{n}^{k}$ be its $k$-th homogeneous component. A linear change of the above basis acts naturally on the spaces $\mathfrak{L}_{n}^{k}$. Denote by $\mathfrak{L}_{n}^{k}(E)$ or simply by $\mathfrak{L}^{k}(E)$ the bundle over $M$ associated with this action. The relation between the characteristic classes of the bundles $E$ and $\mathfrak{L}^{k}(E)$ is described in the following theorem. (This question was already proposed in [Thrall 1942].)

Let $\operatorname{ch}(E) \in H^{*}(M)$ be the Chern character of a bundle $E$. For any element $\eta=\eta_{0}+\eta_{1}+\eta_{2}+\cdots \in H^{*}(M)$ and a number $d$ set $(\eta)_{d}=\eta_{0}+\eta_{1} d+\eta_{2} d^{2}+\cdots$, where $\eta_{i}$ belongs to $H^{2 i}(M)$.

Theorem 2 (see [Reutenauer 1993], for example). The Chern character of the bundle $\mathfrak{L}_{n}^{k}$ is given by

$$
\operatorname{ch}\left(\mathfrak{L}_{n}^{k}\right)=\frac{1}{k} \sum_{d \mid k} \mu(d)\left(\operatorname{ch}(E)^{k / d}\right)_{d} .
$$

Here the summation is taken over the set of all divisors of $k$ and $\mu$ is the Möbius function. By taking the component of degree 0 in this formula we get the wellknown expression

$$
\operatorname{dim}\left(\mathfrak{L}_{n}^{k}\right)=\frac{1}{k} \sum_{d \mid k} \mu(d) n^{k / d} .
$$


for the dimension of $\mathfrak{L}_{n}^{k}$ [Bourbaki 1975].

Proof. The main observation is that the total tensor algebra of $\mathbb{C}^{n}$ is isomorphic, as an $\operatorname{GL}(n, \mathbb{C})$-module, to the universal enveloping algebra of $\mathfrak{L}_{n}$. Therefore, by the Poincaré-Birkhoff-Witt theorem,

$$
T^{*}(E) \cong S^{*}(\mathfrak{L}(E))=S^{*}\left(\mathfrak{L}^{1}\right) \otimes S^{*}\left(\mathfrak{L}^{2}\right) \otimes \cdots .
$$

Applying the Chern character to both sides we get

$$
\frac{1}{1-\operatorname{ch}(E) t}=\prod_{n=1}^{\infty} s\left(\mathfrak{L}^{k}\right)\left(t^{k}\right),
$$

where $t$ is a formal parameter, $s_{i}(V)=\operatorname{ch}\left(S^{i} V\right)$ is the Chern character of the $i$-th symmetric power of $V$, and $s(V)$ is a formal series $s(V)(t)=\sum s_{i}(V) t^{i}$. Now, applying $-t d \log$ to both sides we get

$$
\frac{\operatorname{ch}(E) t}{1-\operatorname{ch}(E) t}=-\sum_{n=1}^{\infty} k t^{k}\left(d \log s\left(\mathfrak{L}^{k}\right)\right)\left(t^{k}\right) .
$$

Observe now that $-d \log s(V)(t)=(\operatorname{ch}(V))_{1}+(\operatorname{ch}(V))_{2} t+(\operatorname{ch}(V))_{3} t^{2}+\cdots$. To prove this we can write (using the splitting principle) $V=V_{1} \oplus \cdots \oplus V_{m}$, where the 1-dimensional bundle $V_{i}$ has Chern character $h_{i}$. Then

$$
\begin{aligned}
-d \log s(V) & =-d \log \left(s\left(V_{1}\right) s\left(V_{2}\right) \ldots s\left(V_{m}\right)\right)=-d \log \prod \frac{1}{1-h_{i} t}=\sum \frac{h_{i}}{1-h_{i} t} \\
& =\sum h_{i}+\sum h_{i}^{2} t+\sum h_{i}^{3} t^{2}+\cdots \\
& =(\operatorname{ch}(V))_{1}+(\operatorname{ch}(V))_{2} t+(\operatorname{ch}(V))_{3} t^{2}+\cdots,
\end{aligned}
$$

since $h_{i}^{k}=e^{k t_{i}}=\sum t_{i}^{l} k^{l} / l$ !, where $t_{i}$ is the first Chern class of $V_{i}$. Therefore, (6) is equivalent to

$$
\frac{\operatorname{ch}(E) t}{1-\operatorname{ch}(E) t}=\sum\left(\operatorname{ch}\left(\mathfrak{L}^{1}\right)\right)_{k} t^{k}+2 \sum\left(\operatorname{ch}\left(\mathfrak{L}^{2}\right)\right)_{k} t^{2 k}+3 \sum\left(\operatorname{ch}\left(\mathfrak{L}^{3}\right)\right)_{k} t^{3 k}+\cdots .
$$

Comparing the terms of the same degree in $t$ we get

$$
\operatorname{ch}(E)^{k}=\sum_{d \mid k} d\left(\operatorname{ch}\left(\mathfrak{L}^{d}\right)\right)_{k / d} .
$$

If we now multiply the $l$-th homogeneous component of this equality by $k^{-l}$ then after this rescaling we get

$$
\left(\operatorname{ch}(E)^{k}\right)_{1 / k}=\sum_{d \mid k} d\left(\operatorname{ch}\left(\mathfrak{L}^{d}\right)\right)_{1 / d} .
$$


Applying to the latter equality the Möbius inversion formula we obtain

$$
k\left(\operatorname{ch}\left(\mathfrak{L}^{k}\right)\right)_{1 / k}=\sum_{d \mid k} \mu(d)\left(\operatorname{ch}(E)^{k / d}\right)_{d / k} .
$$

which (after another rescaling) gives the required formula.

Examples. The relation between the Chern classes and the Chern character gives the possibility to compute the Chern classes of $\mathfrak{L}_{n}^{k}$. For $k \leq 4$ (taking in account only terms of degree at most 4 in the characteristic classes) we obtain the following explicit formulas for the total Chern class of $\mathfrak{L}_{n}^{k}$ :

$$
\begin{aligned}
c\left(\mathfrak{L}_{n}^{1}\right)= & c(E)=1+c_{1}+c_{2}+c_{3}+c_{4}+\cdots \\
c\left(\mathfrak{L}_{n}^{2}\right)= & 1+(-1+n) c_{1}+\left(\left(1-\frac{3}{2} n+\frac{1}{2} n^{2}\right) c_{1}^{2}+(-2+n) c_{2}\right) \\
+ & \left(\left(-1+\frac{11}{6} n-n^{2}+\frac{1}{6} n^{3}\right) c_{1}^{3}+\left(4-4 n+n^{2}\right) c_{1} c_{2}+(-4+n) c_{3}\right) \\
+ & \left(\left(1-\frac{25}{12} n+\frac{35}{24} n^{2}-\frac{5}{12} n^{3}+\frac{1}{24} n^{4}\right) c_{1}^{4}+\left(-6+8 n-\frac{7}{2} n^{2}+\frac{1}{2} n^{3}\right) c_{1}{ }^{2} c_{2}\right. \\
& \left.+\left(3-\frac{5}{2} n+\frac{1}{2} n^{2}\right) c_{2}^{2}+\left(9-6 n+n^{2}\right) c_{1} c_{3}+(-8+n) c_{4}\right)+\cdots \\
c\left(\mathfrak{L}_{n}^{3}\right)= & 1+\left(-1+n^{2}\right) c_{1}+\left(\left(2-n-\frac{3}{2} n^{2}+\frac{1}{2} n^{4}\right) c_{1}^{2}+\left(-3+n^{2}\right) c_{2}\right) \\
+ & \left(\left(-4+3 n+\frac{17}{6} n^{2}-n^{3}-n^{4}+\frac{1}{6} n^{6}\right) c_{1}^{3}+\left(12-4 n-5 n^{2}+n^{4}\right) c_{1} c_{2}+\left(-9+n^{2}\right) c_{3}\right) \\
+ & \left(\left(8-\frac{15}{2} n-\frac{61}{12} n^{2}+\frac{7}{2} n^{3}+\frac{47}{24} n^{4}-\frac{1}{2} n^{5}-\frac{5}{12} n^{6}+\frac{1}{24} n^{8}\right) c_{1}{ }^{4}\right. \\
& +\left(-36+19 n+\frac{35}{2} n^{2}-5 n^{3}-4 n^{4}+\frac{1}{2} n^{6}\right) c_{1}^{2} c_{2} \\
& \left.+\left(18-6 n-\frac{7}{2} n^{2}+\frac{1}{2} n^{4}\right) c_{2}^{2}+\left(36-6 n-11 n^{2}+n^{4}\right) c_{1} c_{3}+\left(-27+n^{2}\right) c_{4}\right)+\cdots \\
c\left(\mathfrak{L}_{n}^{4}\right)= & 1+\left(-n+n^{3}\right) c_{1}+\left(\left(1+n-n^{2}-\frac{1}{2} n^{3}-n^{4}+\frac{1}{2} n^{6}\right) c_{1}^{2}+\left(-2\left(n+n^{3}\right) c_{2}\right)\right. \\
+ & \left(\left(-4-\frac{1}{3} n+2 n^{2}+\frac{8}{3} n^{3}+\frac{3}{2} n^{4}-n^{5}-\frac{1}{2} n^{6}-\frac{1}{2} n^{7}+\frac{1}{6} n^{9}\right) c_{1}^{3}\right. \\
& \left.\quad+\left(8+4 n-4 n^{2}-n^{3}-3 n^{4}+n^{6}\right) c_{1} c_{2}+\left(-4 n+n^{3}\right) c_{3}\right) \\
+ & \left(\left(13-2 n-\frac{77}{12} n^{2}-\frac{35}{4} n^{3}-\frac{3}{4} n^{4}+\frac{5}{2} n^{5}+\frac{55}{24} n^{6}+n^{7}-\frac{1}{2} n^{8}-\frac{1}{4} n^{9}-\frac{1}{6} n^{10}+\frac{1}{24} n^{12}\right) c_{1}{ }^{4}\right. \\
& +\left(-48+12 n^{2}+18 n^{3}+7 n^{4}-5 n^{5}-\frac{3 n^{6}}{2}-2 n^{7}+\frac{1}{2} n^{9}\right) c_{1}{ }^{2} c_{2} \\
& +\left(24+4 n-7 n^{2}-\frac{1}{2} n^{3}-2 n^{4}+\frac{1}{2} n^{6}\right) c_{2}^{2}
\end{aligned}
$$

Remark. Substituting $w_{i}$ for $c_{i}$ in these formulas and reducing coefficients mod 2 one gets the expression for the total Stiefel-Whitney class of $\mathfrak{L}_{n}^{k}$ in the case of a real $n$-dimensional bundle $E$. Note that the coefficients of the polynomials above have integer values for any $n$ and therefore their values mod 2 are well defined. 


\section{Determinantal formula and its application}

Determinantal formula. First we recall a certain formula borrowed from [Fulton 1992]. Assume that we have a flag $A_{1} \subset A_{2} \subset \cdots \subset A_{l}$ of the complex vector bundles over a manifold $M$, with ranks $a_{1} \leq a_{2} \leq \cdots \leq a_{l}$, and a map

$$
h: A_{1} \subset A_{2} \subset \cdots \subset A_{l} \rightarrow B
$$

to a manifold $B$ of dimension $b$. Assume furthermore that the set of nonnegative integers $\kappa_{1}, \ldots, \kappa_{l}$ satisfies the inequalities

$$
0<a_{1}-\kappa_{1}<a_{2}-\kappa_{2}<\cdots<a_{l}-\kappa_{l}, \quad \kappa_{1}<\kappa_{2}<\cdots<\kappa_{l}<b .
$$

Let $\Omega_{\kappa} \subset M$ be the degeneracy locus defined by the conditions $\operatorname{rk}\left(h: A_{i} \rightarrow\right.$ $B) \leq \kappa_{i}, i=1, \ldots, l$, that is, the set of all points $x \in M$ where all the previous conditions are valid. Now consider the Young diagram $\left(p_{1}^{m_{1}}, \ldots, p_{l}^{m_{l}}\right)$ where

$$
\begin{array}{rlrlrl}
p_{1} & =a_{l}-\kappa_{l}, & p_{2} & =a_{l-1}-\kappa_{l-1}, & \ldots, & p_{l}=a_{1}-\kappa_{1}, \\
m_{1} & =b-\kappa_{l}, & m_{2}=\kappa_{l}-\kappa_{l-1}, & \ldots, & m_{l}=\kappa_{2}-\kappa_{1} .
\end{array}
$$

Its dual diagram is $\mu=\left(q_{1}^{n_{1}}, \ldots, q_{l}^{n_{l}}\right)$ where

$$
\begin{array}{lll}
q_{1}=b-\kappa_{1}, & \ldots, & q_{l}=b-\kappa_{l}, \\
n_{1}=a_{1}-\kappa_{1}, & \ldots, & n_{l}=\left(a_{l}-\kappa_{l}\right)-\left(a_{l-1}-\kappa_{l-1}\right) .
\end{array}
$$

Set $c d(\kappa)=|\lambda|, s \lambda=b-\kappa_{1}, s \mu=a_{l}-\kappa_{l}$. Finally, set

$$
\begin{array}{ll}
\rho(i)=\max \left\{s \in[1, l]: i \leq b-\kappa_{s}=m_{1}+\cdots+m_{l+1-s}\right\}, & i=1, \ldots, s \lambda, \\
\rho^{\prime}(i)=\min \left\{s \in[1, l]: i \leq a_{s}-\kappa_{1}=n_{1}+\cdots+n_{s}\right\}, & i=1, \ldots, s \mu .
\end{array}
$$

Proposition 3 (see [Fulton 1992, 10.2]). If the codimension of $\Omega_{r}$ equals $c d(r)$ then the $\mathbb{Z}$-cohomology class $\left[\Omega_{r}\right]_{\mathbb{Z}}$ of $M$ dual to $\Omega_{r}$ is given by

(8) $\left[\Omega_{r}\right]_{\mathbb{Z}}=\operatorname{det}\left(c_{\lambda_{i}-i+j}\left(A_{\rho(i)}^{*}-B^{*}\right)\right)_{1 \leq i, j \leq s \lambda}=\operatorname{det}\left(c_{\mu_{i}-i+j}\left(B-A_{\rho^{\prime}(i)}\right)\right)_{1 \leq i, j \leq s \mu}$,

where* denotes the dual bundle.

Real case. Consider a fixed flag of vector spaces

$$
\mathbb{R}^{a_{1}} \subset \mathbb{R}^{a_{2}} \subset \cdots \subset \mathbb{R}^{a_{l}} .
$$

Denote by $\operatorname{Mat}\left(a_{l}, b\right)$ the space of all linear maps $\mathbb{R}^{a_{l}} \rightarrow \mathbb{R}^{b}$ identified with the space of $\left(a_{l} \times b\right)$-matrices. Two elements $u, v \in \operatorname{Mat}\left(a_{l}, b\right)$ are called equivalent if for all $i=1, \ldots, l$ the restrictions of $u$ and $v$ to $\mathbb{R}^{a_{i}}$ have the same rank. The set of all pairwise equivalent elements in $\operatorname{Mat}\left(a_{l}, b\right)$ will be called a stratum. Obviously, one obtains in this way a finite stratification of $\operatorname{Mat}\left(a_{l}, b\right)$. 
Using the same notation as above consider now a map $h$ of real vector bundles

$$
h: A_{1} \subset A_{2} \subset \cdots \subset A_{l} \rightarrow B .
$$

Let $x \in M$ be a point of the base and $U \ni x$ be its small neighborhood such that the bundles are trivial and trivialized over $U$. Then the map $h$ over $U$ is given by a family of matrices

$$
h_{U}: U \rightarrow \operatorname{Mat}\left(a_{l}, b\right) .
$$

Definition 4. The map $h$ is called transversal at the point $x$ if the map $h_{U}$ is transversal to the stratum containing the point $h_{U}(x)$. The map $h$ is called transversal if it is transversal at every point $x \in M$.

Note that the transversality condition does not depend on the trivialization of the bundles chosen over $U$. Thom's transversality theorem implies that a generic map $h$ is transversal at every point $x$.

Corollary 5. Let $h$ be a transversal map. For any growth vector $r$, its degeneracy locus $\Omega_{r}$ is a closed (possibly empty) subvariety of $M$. The dual $\mathbb{Z}_{2}$-cohomology class given by the intersection index with the smooth part of $\Omega_{r}$ is well-defined and given by the analog of formula (8), with the Chern classes replaced by StiefelWhitney classes.

Proof. One should follow, step by step, the proof of [Fulton 1992, Proposition 10.2]. In fact, one can show that the proof of formula (8) can be reduced to:

(1) The axioms of the Chern classes, the most important of which being the Whitney formula $c(C \oplus F)=c(E) c(F)$.

(2) The isomorphism $H^{*}\left(\mathbb{C} P^{n}\right)=\mathbb{Z}\left[c_{1}\right] / c_{1}^{n+1}$, where $c_{1}$ is the first Chern class of the tautological bundle over $\mathbb{C P}^{n}$.

(3) The construction of the Gysin map $\phi_{*}: H^{*}(X) \rightarrow H^{*}(Y)$ for the proper map $\phi: X \rightarrow Y$ of smooth manifolds, and the Gysin formula $\phi_{*}\left(\phi^{*} a \cup b\right)=$ $a \cup \phi_{*}(b), a \in H^{*}(X), b \in H^{*}(Y)$.

(4) The relation

$$
p_{*}\left(1 /\left(1-c_{1}(S)\right)=c^{-1}\left(E^{*}\right),\right.
$$

where $\pi: E \rightarrow M$ is any complex vector bundle, $p: P \rightarrow M$ is its projectivization, and finally $S$ is the natural tautological linear subbundle in the bundle $p^{*} \pi$ over $P$.

All these elements have real analogs, with $\mathbb{Z}$-cohomology replaced by $\mathbb{Z}_{2}$-cohomology, Chern classes by Stiefel-Whitney classes, $\mathbb{C P}^{n}$ by $\mathbb{R} \mathbb{P}^{n}$ etc. 
Application to subbundles. If we drop the restrictions (7) then for a given nsubbundle $\mathscr{V} \subset T M_{m}$ and a given growth vector $r=\left(r_{1}=n \leq r_{2} \leq \cdots \leq r_{k}=m\right)$ the degeneracy locus $\Sigma_{r}$ is the subset $\Omega_{r} \subset M$ for the map

$$
\Phi_{k}: \mathfrak{L}^{1}(\mathscr{V}) \subset \cdots \subset \bigoplus_{i=1}^{k} \mathfrak{L}^{i}(\mathscr{V}) \rightarrow T M .
$$

Set $L_{j}(\mathscr{V})=\bigoplus_{i=1}^{j} \mathfrak{L}^{i}(\mathscr{V})$ and $\partial(n, j)=\operatorname{dim} \bigoplus_{i=1}^{j} \mathfrak{L}^{i}(\mathscr{V})=\sum_{i=1}^{j} d(n, i)$. To apply Fulton's formula (8) we must get rid of the redundant subspaces, i.e. those subspaces whose rank conditions are automatically satisfied due to the rank conditions imposed on the previous subspaces. (In other words, the $i$-th subspace is redundant if $\partial(n, i)-r_{i}=\partial(n, i-1)-r_{i-1}$. $)$ We define a reduced index set as a maximal subset of indices $I=\left(i_{1}, \ldots, i_{l}\right)$ for which ranks and coranks are both strictly increasing:

$$
r_{i_{1}}<\cdots<r_{i_{l}}<m, 0<\partial\left(n, i_{1}\right)-r_{i_{1}}<\partial\left(n, i_{2}\right)-r_{i_{2}}<\cdots<\partial\left(n, i_{l}\right)-r_{i_{l}} .
$$

One gets the Young diagram $\lambda(r)=\left(p_{1}(r)^{m_{1}(r)}, \ldots, p_{l}(r)^{m_{l}(r)}\right)$, where

$$
\begin{array}{lll}
p_{1}(r)=\partial\left(n, i_{l}\right)-r_{i_{l}}, & p_{2}(r)=\partial\left(n, i_{l-1}\right)-r_{i_{l-1}}, \ldots, & p_{l}(r)=\partial\left(n, i_{1}\right)-r_{i_{1}}, \\
m_{1}(r)=m-r_{i_{l}}, & m_{2}(r)=r_{i_{l}}-r_{i_{l-1}}, & \ldots,
\end{array}
$$

Its dual diagram is $\mu(r)=\left(q_{1}(r)^{n_{1}(r)}, \ldots, q_{l}(r)^{n_{l}(r)}\right)$, where

$$
\begin{aligned}
& q_{1}(r)=m-r_{i_{1}}, \quad \ldots, q_{l}(r)=m-r_{i_{l}}, \\
& n_{1}(r)=\partial\left(n, i_{1}\right)-r_{i_{1}}, \ldots, \quad n_{l}(r)=\left(\partial\left(n, i_{l}\right)-r_{i_{l}}\right)-\left(\partial\left(n, i_{l-1}\right)-r_{i_{l-1}}\right) .
\end{aligned}
$$

Finally, we set $c d(r)=|\lambda(r)|=|\mu(r)|$ is the area of either of these Young diagrams.

Analogously, we have $s \lambda(r)=m-r_{i_{1}}, s \mu(r)=\partial\left(n, i_{l}\right)-r_{i_{l}}$, and

$\rho_{r}(i)=\max \left\{s \in[1, l]: i \leq m-r_{i_{s}}=m_{1}(r)+\cdots+m_{l+1-s}(r)\right\}, \quad i=1, \ldots, s \lambda(r)$, $\rho_{r}^{\prime}(i)=\min \left\{s \in[1, l]: i \leq \partial\left(n, i_{s}\right)-r_{i_{1}}=n_{1}(r)+\cdots+n_{s}(r)\right\}, \quad i=1, \ldots, s \mu(r)$.

Definition 6. The number $c d(r)$ is called the expected codimension of $\Sigma_{r}$.

Main Result. If $\operatorname{codim}\left(\Sigma_{r}(\mathscr{V})\right)$ coincides with the expected codimension $c d(r)$, then the $\mathbb{Z}_{2}$-cohomology class $\left[\Sigma_{r}\right]_{\mathbb{Z}_{2}}$ of the base manifold $M$ dual to $\Sigma_{r}$ is

$$
\begin{aligned}
{\left[\Sigma_{r}\right]_{\mathbb{Z}_{2}} } & =\operatorname{det}\left(w_{\lambda_{i}(r)-i+j}\left(L(\mathscr{V})_{\rho_{r}(i)}^{*}-T M^{*}\right)\right)_{1 \leq i, j \leq s \lambda(r)} \\
& =\operatorname{det}\left(w_{\mu_{i}(r)-i+j}\left(T M-L(\mathscr{V})_{\rho_{r}^{\prime}(i)}\right)\right)_{1 \leq i, j \leq s \mu(r)},
\end{aligned}
$$

where the $w_{l}$ are the Stiefel-Whitney classes.

Examples (compare [Kazarian et al. 1997]). Consider a generic 2-subbundle in $T M_{4}$. There are three possible nonmaximal growth vectors, $(2,2,4),(2,3,3)$, 
and $(2,2,3,4)$. The coincidence of the actual and the expected codimensions in this case follows from the normal forms in [Zhitomirskii 1990]. (In the case $(2,2,2, \ldots)$ the codimension is $\geq 5$.)

Case $r=(2,2,4)$. The reduced index set is $I=\{2\}$, i.e. we have to consider only the map $\Phi_{2}: L_{2}(\mathscr{V}) \rightarrow T M$ of the usual bundles and determine the locus of points where $\operatorname{rk}\left(\Phi_{2}\right) \leq 2$. One has $\operatorname{rk}\left(L_{2}(\mathscr{V})\right)=3, \operatorname{rk}(T M)=4, \lambda(r)=\left(1^{2}\right), s \lambda(r)=2$, $c d(r)=2$. Finally, $\mu(r)=2, s \mu(r)=1$ and $\rho^{\prime}(1)=1$. Therefore,

$$
\left[\Sigma_{(2,2,4)}\right]_{\mathbb{Z}_{2}}=w_{2}\left(T M-L_{2}(\mathscr{V})\right)=w_{2}(M)+w_{2}(\mathscr{V})+w_{1}^{2}(\mathscr{V}) .
$$

Case $r=(2,3,3)$. The reduced index set is $I=\{3\}$, i.e. we have to consider only the map $\Phi_{3}: L_{3}(\mathscr{V}) \rightarrow T M$ of the usual bundles and determine the locus of points where $\operatorname{rk}\left(\Phi_{3}\right) \leq 3$. One has $\operatorname{rk}\left(L_{3}(\mathscr{V})\right)=5, \operatorname{rk}(T M)=4, \lambda(r)=\left(2^{1}\right), s \lambda(r)=1$, $c d(r)=2$. Finally, $\mu(r)=\left(1^{2}\right), s \mu(r)=2$ and $\rho^{\prime}(1)=1, \rho^{\prime}(2)=1$. Therefore,

$$
\begin{aligned}
{\left[\Sigma_{(2,3,3)}\right]_{\mathbb{Z}_{2}} } & =\left|\begin{array}{ll}
w_{1}\left(T M-L_{3}(\mathscr{V})\right) & w_{2}\left(T M-L_{3}(\mathscr{V})\right) \\
w_{0}\left(T M-L_{3}(\mathscr{V})\right) & w_{1}\left(T M-L_{3}(\mathscr{V})\right)
\end{array}\right| \\
& =w_{1}^{2}(M)+w_{1}^{2}(\mathscr{V})+w_{2}(M)+w_{1}(M) w_{1}(\mathscr{V}) .
\end{aligned}
$$

Case $r=(2,2,3,4)$. The reduced index set is $I=\{2,3\}$, so we have to consider the map $\Phi: L_{2}(\mathscr{V}) \subset L_{3}(\mathscr{V}) \rightarrow T M$. One has $\lambda(r)=(2,1), s \lambda(r)=2, c d(r)=3$. Now, $\mu(r)=(2,1), s \mu(r)=2$ and $\rho^{\prime}(1)=1, \rho^{\prime}(2)=2$. Therefore,

$$
\begin{aligned}
{\left[\Sigma_{(2,2,3,4)}\right]_{\mathbb{Z}_{2}} } & =\left|\begin{array}{ll}
w_{2}\left(T M-L_{2}(\mathscr{V})\right) & w_{3}\left(T M-L_{2}(\mathscr{V})\right) \\
w_{0}\left(T M-L_{3}(\mathscr{V})\right) & w_{1}\left(T M-L_{3}(\mathscr{V})\right)
\end{array}\right| \\
& =w_{1}(M) w_{2}(M)+w_{2}(M) w_{1}(\mathscr{V})+w_{1}^{3}(\mathscr{V})+w_{3}(M) .
\end{aligned}
$$

These answers are obtained through standard manipulations with the total Chern class of $T M$ and $\mathfrak{L}_{n}^{k}$.

\section{Transversality property for subbundles and general properties on $f^{\mathcal{V}}$}

To be able to apply formula (11) to subbundles, one needs to show that a certain transversality property is valid for the map $\Phi_{k}: \bigoplus_{i \leq k} \mathfrak{L}^{i}(\mathscr{V}) \rightarrow T M$; see $\S 2$. This condition can be formulated as follows. The total space $\operatorname{Hom}\left(\bigoplus_{i \leq k} \mathfrak{L}^{i}(\mathscr{V}), T M\right)$ has a natural stratification according to different degenerations of the growth vector. The transversality property says that the section of the above bundle determined by the map $\Phi_{k}$ is transversal to each stratum of this natural stratification.

Naturally one wants to know if the transversality property is valid for generic $n$-dimensional subbundles $\mathscr{V} \subset T M$. The conjecture stated below claims that this is indeed the case. (Up to codimension $m-\sqrt{m}$ a similar statement is shown to be valid in [Gershkovich and Vershik 1988].) 
Since the transversality property is essentially local, we formulate it in local terms.

Local problem. Take $M=\mathbb{R}^{m}$ with a fixed system of coordinates $x_{1}, \ldots, x_{m}$ and consider the set $\Omega^{0}$ of germs of $n$-subbundles in $\mathbb{R}^{m}$ such that for any $\mathscr{V} \in \Omega^{0}$ the subspace $\mathscr{V}(0)$ at the origin is spanned by $\partial / \partial x_{1}, \ldots, \partial / \partial x_{n}$. The set $\Omega^{0}$ can be identified with the set of all $n$-tuples of vector-fields $v_{1}, \ldots, v_{n}$ of the form $v_{i}=\partial / \partial x_{i}+\sum_{j=n+1}^{m} a_{i, j}\left(x_{1}, \ldots, x_{m}\right) \partial / \partial x_{j}$. Indeed, fixing the standard Euclidean structure on $\mathbb{R}^{m}$ we can uniquely lift the vector fields $\partial / \partial x_{1}, \ldots, \partial / \partial x_{n}$ to any subbundle $\mathscr{V} \in \Omega^{0}$ and get the $n$-tuple of vector fields $v_{1}(\mathscr{V}), \ldots v_{n}(\mathscr{V})$ with the above properties.

Remark. For each $k$ we have the derived map $\Psi_{k}: \mathscr{V} \rightarrow F l_{k}(\mathscr{V})$ where $F l_{k}(\mathscr{V})=$ $\left(\mathscr{V}=\mathscr{V}_{1} \subset \mathscr{V}_{2} \subset \cdots \subset \mathscr{V}_{k}\right)$. Each $F l_{k}(\mathscr{V})$ is the image under the canonical map of the $\mathscr{A}$-module $N_{k}(\mathscr{V})$, see $\S 2$. Fixing the standard Euclidean structure, the $n$-tuple of vector fields $\partial_{i}$ and the Hall basis we obtain the standard set of sections for all $\mathscr{V} \in \Omega^{0}$ and in all $N_{k}(\mathscr{V})$. This gives us a noncanonical isomorphism between $\mathscr{V}_{1}$ and $\mathscr{V}_{2}$ and between $N_{k}\left(\mathscr{V}_{1}\right)$ and $N_{k}\left(\mathscr{V}_{2}\right)$ as $\mathscr{A}$-modules for any subbundles $\mathscr{V}_{1}, \mathscr{V}_{2}$ and any positive integer $k$. Localizing we can identify the jets of the subbundle $\mathscr{V}$ with the jets of the $n$-tuples of vector fields $v_{1}(\mathscr{V}), \ldots, v_{n}(\mathscr{V})$ and the jets of $F l_{k}(\mathscr{V})$ with the jets of the $\partial(n, k)$-tuples of vector fields obtained from $v_{1}(\mathscr{V}), \ldots, v_{n}(\mathscr{V})$ by applying the commutations prescribed by the elements in the chosen Hall basis. (Recall that $\partial(n, k)=\sum_{i=1}^{k} d(n, i)$.) The map $\Psi_{k}$ induces the well-defined map $\Psi_{k}^{i}: j^{k+i}(\mathscr{V}) \rightarrow j^{i}\left(F l_{k}(\mathscr{V})\right)$ of the corresponding jets.

Remark. The 0 -jet of $F l_{k}(\mathscr{V})$ can be represented by a $m \times \partial(n, i)$-matrix of the form

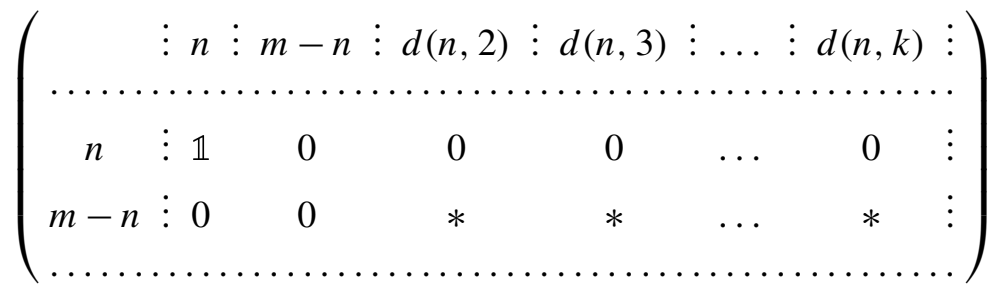

Here $\mathbb{1}$ and 0 denote the identity and the zero matrices of the sizes given in the first row and the first column and ${ }^{\prime} *^{\prime}$ stands for arbitrary real entries.

Notation. Let $\operatorname{Mat}^{0}(n, m, k)$ denote the subset of all $m \times \partial(n, k)$-matrices of the above form (12) and $J(n, m, k)$ denote the space of $k$-jets of $\mathscr{V} \in \Omega^{0}$. The space $J(n, m, k)$ is isomorphic to the space of all $n(m-n)$-tuples of polynomials in $m$ variables of degree $\leq k$, see above. Obviously, $\operatorname{dim} J(n, m, k)=(m-n) n\left(\begin{array}{c}m+k \\ k\end{array}\right)$ and $\operatorname{dim} \operatorname{Mat}^{0}(n, m, k)=(m-n) \sum_{i=2}^{k} d(n, i)$. 
The main object of the remaining part of the paper is the polynomial map of affine spaces

$$
\Psi_{k}^{0}: J(n, m, k-1) \rightarrow \operatorname{Mat}^{0}(n, m, k) .
$$

The space $\operatorname{Mat}^{0}(n, m, k)$ has the following natural stratification. We start with the obvious inclusions $\operatorname{Mat}^{0}(n, m, 1) \subset \operatorname{Mat}^{0}(n, m, 2) \subset \cdots \subset \operatorname{Mat}^{0}(n, m, k)$. Now fixing the growth vector $r=\left(r_{1}=n \leq r_{2} \leq \cdots \leq r_{k} \leq \partial(n, k)\right)$ we define the subset $\operatorname{Mat}_{r}^{0}(n, m, k) \subset \operatorname{Mat}^{0}(n, m, k)$ of all matrices whose restrictions to $\operatorname{Mat}^{0}(n, m, i)$ have rank $\leq r_{i}$ for all $i=1, \ldots, k$. Rather obviously, the codimension of $\operatorname{Mat}_{r}^{0}(n, m, k)$ in $\operatorname{Mat}^{0}(n, m, k)$ equals $c d(r)$.

Finally we are in position to formulate the required transversality property.

Main Conjecture. The subset of points $x$ in $J(n, m, k)$ such that the map $\Psi_{k}^{0}$ is nontransversal to the stratum containing the point $\Psi_{k}^{0}(x) \in \operatorname{Mat}^{0}(n, m, k)$ has codimension in $J(n, m, k)$ strictly exceeding $m$.

Thom's transversality theorem implies that the validity of the above conjecture immediately leads to the transversality assumption for generic $n$-dimensional subbundles in $T M$.

We were unable to prove the above conjecture in its complete generality but we were able to settle a number of cases given below.

Transversality Theorem. The required transversality property holds either for $n \geq 3$ and $m \leq \frac{1}{6} n(n+1)(2 n+1)=\partial(n, 3)$ or for $n=2$ and $m \leq 8=\partial(2,4)$. Namely, for $k=2,3$ and any $m \geq n$ the map $\Psi_{k}^{0}$ is a submersion. Also, for $n=2$ and any $m \geq n$ and $k=4$ the map $\Psi_{4}^{0}$ is a submersion.

Violation of transversality for big codimensions. We will finish this section by pointing out that the behavior of the image $\Psi_{k}^{0}(J(n, m, k-1))$ w.r.t the natural rank stratification of the space $\operatorname{Mat}^{0}(n, m, k)$ is highly nontrivial. The next two statements show that one can only hope that transversality holds for the strata of relatively small codimension, as stated in the main conjecture.

Lemma 7. For any fixed $m \geq n$ and for $k>$ const $\left.\log _{\left(\begin{array}{c}n \\ 2\end{array}\right)}\left(\begin{array}{c}2 m \\ m\end{array}\right)\right)$, one has

$$
\operatorname{dim} J(n, m, k-1)<\operatorname{dim}_{\operatorname{Mat}^{0}}(n, m, k),
$$

and therefore $\Psi_{k}$ is not surjective.

Proof. This is simply dimension count since

$$
\operatorname{dim} J(n, m, k-1)=(m-n) n\left(\begin{array}{c}
m+k-1 \\
k-1
\end{array}\right)
$$

and $\operatorname{dim} \operatorname{Mat}^{0}(n, m, k)=(m-n) \sum_{i=2}^{k} d(n, i)$ where $k d(n, k)=\sum_{j \mid k} \mu(j) n^{k / j}$, where $\mu(j)$ denotes the Möbius function. 
Lemma 8. For any $m \geq n \geq 3$ and $k \geq 4$ the map $\Psi_{k}$ is never onto. The same holds for $n=2$ and $k \geq 5$.

Proof. Consider a matrix in $\mathrm{Mat}^{0}(n, m, 4)$ of the form

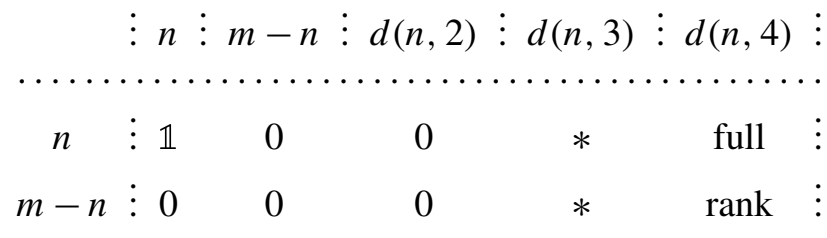

Such a matrix is never in the image of $\Psi_{4}$ since the fourth homogeneous component $H^{4}$ of the Hall basis contains elements of the form $\left(\left(v_{i}, v_{j}\right),\left(v_{p}, v_{q}\right)\right)$ which vanish since the first commutators of all basic vector fields vanish. (For $n=2$ the same effect happens for $k \geq 5$.)

\section{Transversality theorem and defect vectors}

This section is devoted to the proof of transversality theorem of the previous section as well as to the description of generic defect vectors in our situation. Namely, let $w=\left(\left(\left(v_{i_{1}}, v_{i_{2}}\right)\left(v_{i_{3}}, v_{i_{4}}\right)\right) \ldots\left(\left(v_{i_{k-4}}, v_{i_{k-3}}\right)\left(v_{i_{k-1}}, v_{i_{k}}\right)\right)\right) \in H^{k}$ be any element of length $k$ in the Hall basis.

Definition 9. By the depth $d p_{w}\left(v_{i_{j}}\right)$ of any variable $v_{i_{j}}$ in $w$ we denote the difference between the number of opening and closing parentheses preceding $v_{i_{j}}$. By the depth $d p(w)$ of $w$ we mean $\max _{j} d p_{w}\left(v_{i_{j}}\right)$.

Obviously, for any $j$ one has $d p_{w}\left(v_{i_{j}}\right) \leq d p(w)$ and there exist at least 2 different values of $j$ for which $d p_{w}\left(v_{i_{j}}\right)=d p(w)$.

Let $H^{p}$ denote the $p$-th homogeneous component of $H$ and $H^{p, q}$ be its subset containing all elements of depth $q$. Obviously, $q$ can vary between $\left[\log _{2}(p)\right]+1$ and $p$. We restrict the map $\Psi_{k}^{0}$ to the union $\bigcup_{j=1}^{k} H^{j, j}$.

Lemma 10. For any $k$ and $m \geq n$ the restricted map

$$
\Psi_{k}^{\mathrm{res}}: J(n, m, k-1) \rightarrow \operatorname{Mat}_{\mathrm{res}}^{0}(n, m, k)
$$

is a submersion where $\operatorname{Mat}_{\mathrm{res}}^{0}(n, m, k) \subset \operatorname{Mat}^{0}(n, m, k)$ contains only rows corresponding to the elements from $\bigcup_{j=1}^{k} H^{j, j}$.

Proof. We use induction on $k$.

Base of induction: $k=2$. One has $\left(v_{i}, v_{j}\right)_{l}=a_{j, i, l}-a_{i, j, l}$ where $\left(v_{i}, v_{j}\right)_{l}$ is the value of the $l$-th component of the commutator $\left(v_{i}, v_{j}\right)$ at the origin, $a_{j, i, l}$ is the coefficient at $x_{i}$ in the $l$-th component of $v_{j}$ and $a_{i, j, l}$ is the coefficient at $x_{j}$ in 
the $l$-th component of $v_{i}$. Since $a_{j, i, l}$ and $a_{i, j, l}$ are independent parameters in the preimage the map from $J(n, m, 1)$ to $\operatorname{Mat}_{\mathrm{res}}^{0}(n, m, 2)$ is, obviously, a submersion. Induction step. Assume the statement is proved for $j<k$. Elements in $H^{k, k}$ are in one-to-one correspondence with the commutators $\left(v_{i_{1}}\left(v_{i_{2}}\left(\ldots\left(v_{i_{k-1}}, v_{i_{k}}\right)\right) \ldots\right)\right.$, where $i_{1} \geq i_{2} \geq \cdots \geq i_{k-1}<i_{k}$; see the definition of the Hall basis. In particular, the number $\#_{k}(n)$ of such elements equals $\sum_{j=0}^{n-1}\left(\begin{array}{c}j+k-2 \\ j\end{array}\right) j$. For example,

$$
\begin{gathered}
\#_{2}(n)=\frac{1}{2} n(n-1), \quad \#_{4}(n)=\frac{1}{8}(n-1) n(n+1)(n+2), \\
\#_{3}(n)=\frac{1}{3} n(n-1)(n+1), \quad \#_{5}(n)=\frac{1}{30} n(n-1)^{2}(n+2)(n+3), \\
\#_{6}(n)=\frac{1}{16} n^{2}\left(n^{2}-1\right)\left(n^{2}+n+2\right) .
\end{gathered}
$$

One has

$$
\left(v_{i_{1}}\left(v_{i_{2}}\left(\ldots\left(v_{i_{k-1}}, v_{i_{k}}\right) \ldots\right)\right)\right)_{l}=\text { const }+\frac{a_{i_{k}, i_{1} i_{2} \ldots i_{k-1}, l}}{c\left(i_{1}, \ldots, i_{k-1}\right)}-\frac{a_{i_{k-1}, i_{1} i_{2} \ldots i_{k-2} i_{k}, l}}{c\left(i_{1}, \ldots, i_{k-2}, i_{k}\right)} .
$$

Here $\left(v_{i_{1}}\left(v_{i_{2}}\left(\ldots\left(v_{i_{k-1}}, v_{i_{k}}\right) \ldots\right)\right)\right)_{l}$ is the value at 0 of the $l$-th component of the commutator vector field corresponding to the element of $H^{k, k}$ under consideration. The constant summand on the right depends only on the $(k-2)$-jet of the basic vector fields $v_{1}(\mathscr{V}), \ldots, v_{n}(\mathscr{V})$, and $c\left(i_{1}, \ldots, i_{k-1}\right), c\left(i_{1}, \ldots, i_{k-2}, i_{k}\right)$ are certain combinatorial constants. Finally, $a_{i_{k}, i_{1} i_{2} \ldots i_{k-1}, l}$ denotes the variable coefficient at the product $x_{i_{1}} x_{i_{2}} \ldots x_{i_{k-1}}$ in the $l$-th component of the basic vector field $v_{i_{k}}$, and $a_{i_{k-1}, i_{1} i_{2} \ldots i_{k-2} i_{k}, l}$ denotes the coefficient at the product $x_{i_{1}} x_{i_{2}} \ldots x_{i_{k-2}} x_{i_{k}}$ in the $l$-th component of $v_{i_{k-1}}$. One can easily check that $a_{i_{k}, i_{1} i_{2} \ldots i_{k-1}, l}$ appears only in the commutator $\left(v_{i_{1}}\left(v_{i_{2}}\left(\ldots\left(v_{i_{k-1}}, v_{i_{k}}\right) \ldots\right)\right)\right)_{l}$. Since all these variable coefficients are independent parameters in the preimage, we get a submersion of the space $\Omega_{\mathfrak{J}}$ on the space $\widetilde{\text { Mat }} t_{\text {res }}^{0}(n, m, k)$. Here $\Omega_{\mathfrak{J}}$ is the subspace of all subbundles in $\Omega$ with some arbitrary fixed $(k-2)$-jet $\mathfrak{J}$ and $\widetilde{\mathrm{Mat}}_{\text {res }}^{0}(n, m, k)$ is the last block in $\operatorname{Mat}_{\mathrm{res}}^{0}(n, m, k)$.

Corollary 11. Lemma 10 implies the Transversality Theorem (page 247).

Proof. Indeed, if $n=2, m \leq 8$ or $n \geq 3, m \leq \frac{1}{6} n(n+1)(2 n+1)=\partial(n, 3)$, one has $H^{k}=H^{k, k}$.

Description of generic defect vectors. We conclude this section with a conjectural description of all degenerations of the associated flag bundle $f \mathscr{V}$ that might occur for a generic $n$-dimensional bundle $\mathscr{V}$ in $T M$.

Definition 12. For any growth vector $r=\left(r_{1}=n, \ldots, r_{k}=m\right)$, the defect vector of $r$ is

$$
\left(\partial(n, 1)-r_{1}=0 ;\left(\partial(n, 2)-r_{2}\right)-\left(\partial(n, 1)-r_{1}\right) ; \ldots ;\right.
$$

$$
\left.\left(\partial(n, k-1)-r_{k-1}\right)-\left(\partial(n, k-2)-r_{k-2}\right) ; 0\right) .
$$


(Up to a reduction of redundant indices this definition coincides with that of the $n_{i}$ in (10).) A stratum $\mathrm{St}_{r}$ is called admissible if codimSt $r \leq m$, and potentially admissible if $c d(r) \leq m$. Next, $\mathrm{St}_{r}$ is called bounding (potentially bounding) if it is nonadmissible (potentially nonadmissible) and is not contained in the closure of any nonadmissible (potentially nonadmissible) stratum distinct from $\mathrm{St}_{r}$.

Lemma 13. For any $n \geq 3$ and any $k \geq 1$ one has $d(n, k+1)>\partial(n, k)=$ $\sum_{j=1}^{k} d(n, j)$. For $n=2$ and any $k \geq 1$ one has $d(2, k+1)+d(2, k+2)>$ $\partial(2, k)=\sum_{j=1}^{k} d(2, j)$.

Proof. We will consider only the case $n \geq 3$. We construct for each element $a \in H^{j}, j \leq k$ the unique element in $H^{k+1}$, i.e. embed $\bigcup H_{j=1, \ldots, k}^{j}$ into $H^{k+1}$, thus proving that $d(n, k+1)>\partial(n, k)$. Recall that the Hall family is linearly ordered and each element in the Hall family has a unique representation in the form $(a(b c))$ where $a, b, c$ satisfy the conditions: $a, b, c, b c$ belong to $H, a \geq b$ and $b<c$. For all $j<\left[\frac{k+1}{2}\right]$ we associate to any element $a \in H^{j}$ the element $\left(a\left(v_{1}\left(v_{1}\left(\ldots\left(v_{1}, v_{2}\right) \ldots\right)\right)\right)\right) \in H^{k+1}$. Now let $j>\left[\frac{k+1}{2}\right]$ and $a$ be some element in $H^{j}$. Then $a=(f, g)$ where $f<g$. Assume additionally that $2 \operatorname{lng}(f)+\operatorname{lng}(g) \leq$ $k+1$. Then we associate to $a$ the element $(h(f, g))$ where $h$ is the maximal element in $H^{k+1-\operatorname{lng}(h)-\operatorname{lng}(g)}$. (Note that, by definition, $h \geq f$.) If $2 \operatorname{lng}(f)+\operatorname{lng}(g)>$ $k+1$ then we choose for each $a=(f, g) \in H^{j}$ the element $(f(h, g))$ where $h$ is the minimal element in $H^{k+1-\operatorname{lng}(h)-\operatorname{lng}(g)}$. The last case to consider is $H^{l}$ when $k=2 l-1$. One has that $\Lambda^{2}\left(H^{l}\right)$ is embedded into $H^{2 k}$ and under the assumption $n \geq 3$ one has $\operatorname{dim} \Lambda^{2}\left(H^{l}\right) \geq \operatorname{dim} H^{l}$. Combining all choices together we obtain a set-theoretic embedding of $\bigcup_{j=1}^{k} H^{j}$ into $H^{k+1}$. The result follows. More detailed consideration shows that $d(2, k+1)+d(2, k+2)>\partial(2, k)$.

Remark. The transversality property is equivalent to showing that the codimension of each potentially admissible (resp. potentially bounding) stratum $\mathrm{St}_{r}$ equals $c d(r)$ and therefore $\mathrm{St}_{r}$ is, in fact, admissible (resp. bounding).

We now list the defect vectors of all potentially admissible strata.

Lemma 14. For $n \geq 3$ the defect vectors of all potentially admissible strata are as in one of the following cases. We assume that $\partial(n, p) \leq m<\partial(n, p+1)$.

(a) There is a 1 in position $l<p, a \chi$ in position $p$, and 0 in all other positions, with the further restrictions $(m-\partial(n, p)+1+\chi) \chi \leq \partial_{l}-1$ and $\chi \geq 0$.

(b) There is a 1 in position $l<p, a \chi$ in position $p+1$, and 0 in all other positions, with the further restrictions $(m-\partial(n, p+1)+1+\chi) \chi \leq \partial_{l}-1$ and $\chi+1+$ $m-\partial(n, p+1) \geq 0$.

(c) There is $a \chi$ in position $p, a v$ in position $p+1$, and 0 in all other positions, with the further restrictions $(m-\partial(n, p)+\chi) \chi+(m-\partial(n, p+1)+\chi+v) v \leq m$ and $m-\partial(n, p+1)+\chi+v \geq 0$. 
Proof. If the rank of the image of some $L_{l}(\mathscr{V})=\bigoplus_{i=1}^{l} \mathfrak{L}^{i}(\mathscr{V})$, where $l<p$, drops then it can drop exactly by 1 . Indeed, assume that it drops by at least 2 then using (10) we get for the dual diagram $\mu_{r}$ that $q_{1} \geq m-\partial(n, l)+2$ and $n_{1} \geq 2$. But, by Lemma 13 one has $2(m-\partial(n, l)+2)>m$ which contradicts the assumption $c d(r) \leq m$. Analogously, if $L_{l}(\mathscr{V})$, with $l<p$, has corank 1 , the corank can possibly increase again only for either $L_{p}(\mathscr{V})$ or $L_{p+1}(\mathscr{V})$; see description below. Indeed, assume that the corank drops by 1 at $L_{l_{1}}(\mathscr{V})$ and by 2 at $L_{l_{2}}(\mathscr{V})$ where $l_{1}<l_{2}<k$. Then by (10) one has $q_{1}=m-\partial\left(n, l_{1}\right)+1, n_{1}=1, q_{2}=m-\partial\left(n, l_{2}\right)+2, n_{2}=1$. But, again by Lemma 13, one gets $q_{1}+q_{2}>m$, which contradicts $c d(r) \leq m$. Therefore the second rank drop can only occur either at position $p$ or $p+1$. If we have that $L_{l}(\mathscr{V}), l<p$ has corank 1 then further corank drops at both positions $p$ and $p+1$ are simultaneously impossible, i.e., only one extra drop is allowed. Indeed, assume that we have coranks $1,2,3$ at positions $l, p, p+1$ respectively. Then by (10) one gets $q_{1}=m-\partial(n, l)+1, n_{1}=1 ; q_{2}=m-\partial(n, p)+2, n_{2}=1$; $q_{3}=1, n_{3}=\partial(n, p+1)-m-1$. But $q_{2} n_{2}+q_{3} n_{3}=m-\partial(n, p)+2+\partial(n, p+$ $1)-m-1=d(n, p)+1$. Again, by Lemma 13 one gets $q_{1} n_{1}+q_{2} n_{2}+q_{3} n_{3}>m$. Thus if the rank drops in some position prior to $p$ we are left with cases (a) and (b). The above list of inequalities follows from the expressions for the terms in the dual Young diagram $\mu=\left(q_{1}^{n_{1}}, q_{2}^{n_{2}}\right)$ given below. In case (a) one gets $q_{1}=m-\partial(n, l)+1$, $n_{1}=1, q_{2}=m-(\partial(n, p)-1-\chi), n_{2}=\chi$. In case (b) one gets $q_{1}=m-\partial(n, l)+1$, $n_{1}=1, q_{2}=m-(\partial(n, p+1)-1-\chi), n_{2}=\chi$. Finally, in case (c) one gets

$$
q_{1}=m-\partial(n, p)+\chi, n_{1}=\chi, \quad q_{2}=m-\partial(n, p+1)+\chi+v, n_{2}=v .
$$

The inequalities express the condition $c d(r) \leq m$ and the condition that the second rank drop actually occurs. One can easily show that (a) are (b) are mutually exclusive: for a given pair $(n, m)$ either the inequalities for (a) or those for (b) can be satisfied under the assumption that $\chi>0$.

Corollary 15. The defect vectors for all potentially bounding strata have one of the forms given in the table.

$$
\begin{array}{lllllllllllllll}
\vdots & 1 & 2 & \ldots & l_{1}-1 & l_{1} & l_{1}+1 & \ldots & l_{2}-1 & l_{2} & l_{2}+1 & \ldots & p-1 & p & p+1
\end{array} \vdots
$$

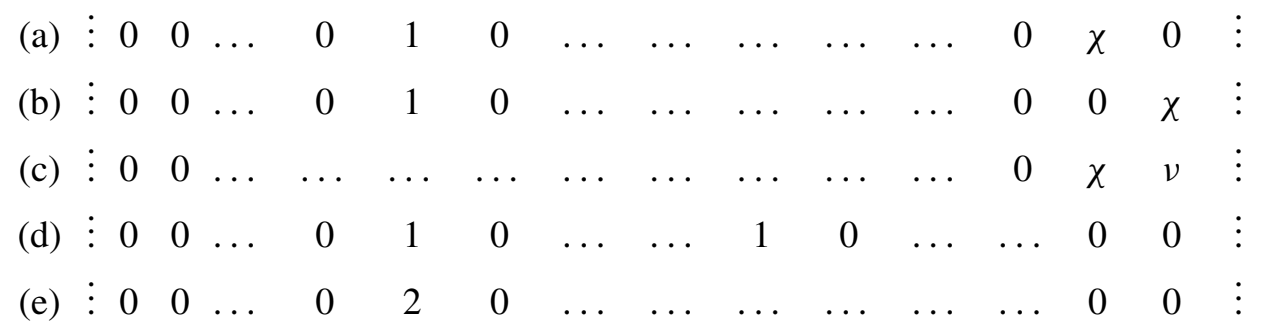


In cases (a)-(c), whose form coincides with that in Lemma 14, we additionally require that the inequalities from the formulation of Lemma 14 are violated for the considered values of $v$ and $\chi$ but satisfied for any smaller nonnegative values of these variables.

Proof. Obtained by a simple case-by-case consideration.

\section{Final remarks}

1. The basic problem related to the above topological obstructions is to what extent vanishing of these obstructions guarantees the existence of a maximal growth subbundle. The result of T. Vogel on the existence of Engel structures on parallelizable 4-manifolds brings a certain amount of optimism about this problem. For example:

Problem. Does every closed parallelizable m-dimensional manifold admits a maximal growth distribution of rank $1<n<m$ ?

2. The theory developed here is incomplete since we lack a proof of the transversality conjecture. Essentially, to accomplish the proof one should only consider the defect vectors listed at the end of Section 6. The authors are convinced that progress in this direction will be intimately related to a detailed study of combinatorics of different Hall bases.

3. The natural representation of $\mathrm{GL}_{n}(\mathbb{C})$ in $\mathfrak{L}_{n}^{i}$ was extensively studied in the series of papers [Thrall 1942; Wever 1949; Klyachko 1974] and some others. There exists an interesting formula for the multiplicity of each irreducible representation (corresponding to some Young diagram $\mu$ ) in $\mathfrak{L}_{n}^{i}$ analogous to the one for the dimension of $\mathfrak{L}_{n}^{i}$. This suggests the existence of a much more sophisticated theory of characteristic classes for subbundles in the tangent bundle since there exist many different natural filtrations in $\mathfrak{L}_{n}$ the most complicated of which coming from the direct sum of irreducible $\mathrm{GL}_{n}(\mathbb{C})$-representations. The first step in this direction will be to find analogs of Theorem 2 for these other filtrations; the second, to find the analogous transversality theorems. As an example of characteristic classes different from the ones considered above one can try to calculate the characteristic classes related to the depth filtration of $\mathfrak{L}_{n}$ introduced in Section 6. For this case there exists a character formula in terms of plethysms analogous to (5) suggested to the authors by C. Reutenauer but it is unfortunately rather complicated.

4. Another natural question related to the transversality theorem is to understand for strata of what corank in the space $\operatorname{Mat}^{0}(n, m, k)$ it holds and therefore to extend the determinantal formulas to the case of nongeneric subbundles or families of subbundles.

Problem. Generalize the transversality theory to the case of nongeneric subbundles. 


\section{Appendix}

Here is a Mathematica program for the calculation of Chern classes of homogeneous components $\mathfrak{L}_{n}^{k}$ of free Lie algebra bundles up to a given order. order $=4$;

(* This function computes the Chern character via the total Chern class

classtochar [cc_] := (resc [n-t D $\left.[\log [c c], t],-1] / . t^{\wedge} k_{-}->t \wedge k / k !\right)$ $+0[\mathrm{t}]^{\wedge}$ ( order +1$) / /$ ExpandAll;

(* This function calculates total Chern class via Chern character

chartoclass [ch_] :=Exp [-Integrate [PolynomialQuotient [resc $[\mathrm{ch},-1] /$.

$\mathrm{t}^{\wedge} \mathrm{k}_{-}->\mathrm{t}^{\wedge} \mathrm{k} \mathrm{k}$ ! $\left.\left.\left., \mathrm{t}, \mathrm{t}\right], \mathrm{t}\right]+0[\mathrm{t}]^{\wedge}(\operatorname{order}+1)\right] / /$ ExpandAll;

(* This function makes the rescaling $\eta \rightarrow(\eta)_{l}$.

$\operatorname{resc}\left[\right.$ eta $\left._{-}, I_{-}\right]:=$Normal [eta]/.t->1 t;

(* Total Chern class of original bundle.

class $=1+$ Sum $\left[c[i] t^{\wedge} i, i\right.$, order $]+0[t]^{\wedge}($ order +1$)$;

(* Chern character of original bundle.

char=classtochar [class];

(* Computes the Chern character of $\mathfrak{L}^{\wedge} k$ using Theorem 2.

charofL $\left[k_{-}\right]:=\left(\right.$Plus@@ $\left(\right.$MoebiusMu $\left.[\#] \operatorname{resc}\left[\operatorname{char}^{-}(\mathrm{k} / \#), \#\right] / \mathrm{k}\right) \& / @$

$($ Divisors $[\mathrm{k}]))) / /$ ExpandAll;

(* Total Chern class of $\mathfrak{L}^{\wedge} k$ as a series in $t$

classofL[k_]:=chartoclass [charofL $[\mathrm{k}]]$;

\section{Acknowledgments}

The starting point of this article was the special case of 2-subbundles on $M_{4}$ considered in [Kazarian et al. 1997]. Later the authors realized that analogous computations over $\mathbb{Z}_{2}$ can be carried out in the general setup described in the present paper. Sincere thanks are due to R. Montgomery for important discussions of the subject and numerous remarks which substantially improved the quality of exposition. Kazarian is grateful to IHES (Paris), MPIM (Bonn) and MSRI (Berkeley), where parts of this project were carried out.

\section{References}

[Bourbaki 1975] N. Bourbaki, Lie groups and Lie algebras, Chapters 1-3, Addison-Wesley, Reading, MA, 1975. Reprinted Springer, Berlin, 1989. MR 2001g:17006 Zbl 0672.22001

[Fulton 1984] W. Fulton, Intersection theory, Ergebnisse der Math. (3) 2, Springer, Berlin, 1984. MR 85k:14004 Zbl 0541.14005 
[Fulton 1992] W. Fulton, "Flags, Schubert polynomials, degeneracy loci, and determinantal formulas”, Duke Math. J. 65:3 (1992), 381-420. MR 93e:14007 Zbl 0788.14044

[Fulton and Pragacz 1998] W. Fulton and P. Pragacz, Schubert varieties and degeneracy loci, Lecture Notes in Mathematics 1689, Springer, Berlin, 1998. MR 99m:14092 Zbl 0913.14016

[Gershkovich 1988] V. Y. Gershkovich, "On normal form of distribution jets”, pp. 77-98 in Topology and geometry-Rohlin Seminar, Lecture Notes in Mathematics 1346, Springer, Berlin, 1988. MR 90e:58004 Zbl 0649.58004

[Gershkovich 1995a] V. Gershkovich, "Exotic Engel structures on $\mathbf{R}^{4}$ ", Russian J. Math. Phys. 3:2 (1995), 207-226. MR 96i:53036 Zbl 0909.53022

[Gershkovich 1995b] V. Gershkovich, “On simplest Engel structures on 4-manifolds”, pp. 279-294 in Dynamical systems and applications, edited by R. P. Agarwal, World Sci. Ser. Appl. Anal. 4, World Sci., River Edge, NJ, 1995. MR 96k:58010 Zbl 0857.57029

[Gershkovich and Vershik 1988] V. Gershkovich and A. Vershik, "Nonholonomic manifolds and nilpotent analysis", J. Geom. Phys. 5:3 (1988), 407-452. MR 91j:58011 Zbl 0693.53006

[Hirzebruch and Hopf 1958] F. Hirzebruch and H. Hopf, "Felder von Flächenelementen in 4-dimensionalen Mannigfaltigkeiten”, Math. Ann. 136 (1958), 156-172. MR 20 \#7272 Zbl 0088.39403

[Jacobowitz and Mendoza 2003] H. Jacobowitz and G. Mendoza, "Sub-bundles of the complexified tangent bundle", Trans. Amer. Math. Soc. 355:10 (2003), 4201-4222. MR 2005a:58030 Zbl 1031.58012

[Kazarian et al. 1997] M. Kazarian, R. Montgomery, and B. Shapiro, "Characteristic classes for the degenerations of two-plane fields in four dimensions", Pacific J. Math. 179:2 (1997), 355-370. MR 98d:57046 Zbl 0895.58004

[Klyachko 1974] A. A. Klyachko, "Lie elements in the tensor algebra", Sib. Mat. Zh. 15 (1974), 1296-1304. In Russian; translated in Siberian Math. J. 15 (1974), 914-920. Zbl 0315.15015

[Laksov 1994] D. Laksov, "Remarks on Giovanni Zeno Giambelli’s work and life", Rend. Circ. Mat. Palermo (2) Suppl. 36 (1994), 207-218. MR 1308346 Zbl 0926.01007

[Matsushita 1988] Y. Matsushita, "Fields of 2-planes on compact simply-connected smooth 4-manifolds”, Math. Ann. 280:4 (1988), 687-689. MR 89i:57013 Zbl 0628.57016

[Montgomery 2002] R. Montgomery, A tour of subriemannian geometries, their geodesics and applications, Mathematical Surveys and Monographs 91, American Mathematical Society, Providence, RI, 2002. MR 2002m:53045 Zbl 1044.53022

[Reutenauer 1993] C. Reutenauer, Free Lie algebras, London Math. Soc. Monographs N.S. 7, Oxford Univ. Press, New York, 1993. MR 94j:17002 Zbl 0798.17001

[Thomas 1967a] E. Thomas, "Fields of tangent 2-planes on even-dimensional manifolds", Ann. of Math. (2) 86 (1967), 349-361. MR 35 \#3699 Zbl 0168.21401

[Thomas 1967b] E. Thomas, "Fields of tangent $k$-planes on manifolds", Invent. Math. 3 (1967), 334-347. MR 36 \#903 Zbl 0162.55402

[Thomas 1969] E. Thomas, "Vector fields on manifolds", Bull. Amer. Math. Soc. 75 (1969), 643683. MR 39 \#3522 Zbl 0183.51703

[Thrall 1942] R. M. Thrall, "On symmetrized Kronecker powers and the structure of the free Lie ring”, Amer. J. Math. 64 (1942), 371-388. MR 3,262d Zbl 0061.04201

[Vogel 2004] T. Vogel, "Existence of Engel structures”, preprint, 2004. math.GT/0411217

[Vogel 2005] T. Vogel, "A construction of Engel structures", C. R. Math. Acad. Sci. Paris 340:1 (2005), 43-48. MR 2005j:58002 Zbl 1062.58006 
[Wever 1949] F. Wever, "Über Invarianten in Lieschen Ringen”, Math. Ann. 120 (1949), 563-580. MR 10,676e Zbl 0032.10701

[Zhitomirskii 1990] M. Y. Zhitomirskiü, "Normal forms of germs of two-dimensional distributions on $\mathbb{R}^{4}$ ", Funktsional. Anal. i Prilozhen. 24:2 (1990), 81-82. In Russian; translated in Funct. Anal. Appl. 24:2 (1990), 150-152. MR 91g:58011 Zbl 0711.58001

Received May 13, 2005. Revised August 10, 2005.

BORIS SHAPIRO

DEPARTMENT OF MATHEMATICS

STOCKHOLM UNIVERSITY

SE-106 91, STOCKHOLM

SWEDEN

shapiro@math.su.se

MAXIM KAZARIAN

STEKLOV MATHEMATICAL INSTITUTE

42 VAVILOVA ST.

117966, Moscow GSP-1

RUSSIA

kazarian@mccme.ru 